\title{
Quasifission in heavy and superheavy element formation reactions
}

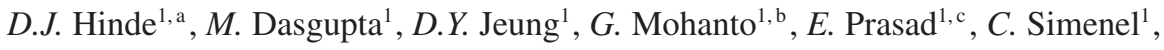 \\ J. Walshe ${ }^{1}, A$. Wahkle ${ }^{1, \mathrm{~d}}$, E. Williams ${ }^{1}$, I.P. Carter ${ }^{1}$, K.J. Cook $^{1}$, Sunil Kalkal ${ }^{1}$, \\ D.C. Rafferty ${ }^{1}$, R. du Rietz ${ }^{1, \mathrm{e}}$, E.C. Simpson ${ }^{1}$, H.M. David ${ }^{2}$, Ch.E. Düllmann ${ }^{2,3,4}$, \\ and $J$. Khuyagbaatar ${ }^{2,3}$ \\ ${ }^{1}$ Department of Nuclear Physics, Research School of Physics and Engineering, Australian National \\ University, Canberra, ACT 2601, Australia \\ ${ }^{2}$ GSI Helmholtzzentrum für Schwerionenforschung, 64291 Darmstadt, Germany \\ ${ }^{3}$ Helmholtz Institute Mainz, 55099 Mainz, Germany \\ ${ }^{4}$ Johannes Gutenberg Universität Mainz, 55099 Mainz, Germany
}

\begin{abstract}
Superheavy elements are created in the laboratory by the fusion of two heavy nuclei. The large Coulomb repulsion that makes superheavy elements decay also makes the fusion process that forms them very unlikely. Instead, after sticking together for a short time, the two nuclei usually come apart, in a process called quasifission. Mass-angle distributions give the most direct information on the characteristics and time scales of quasifission. A systematic study of carefully chosen mass-angle distributions has provided information on the global trends of quasifission. Large deviations from these systematics reveal the major role played by the nuclear structure of the two colliding nuclei in determining the reaction outcome, and thus implicitly in hindering or favouring superheavy element production.
\end{abstract}

Superheavy elements (SHE) are formed by heavy-ion fusion reactions. Their cross sections are significantly suppressed [1] by the quasifission process [2]. This non-equilibrium process results when the combined di-nuclear system, formed initially when the two nuclear surfaces stick together, subsequently separates into two (fission-like) fragments. This occurs very rapidly, typically $\sim 10^{-20} \mathrm{~s}$, well-before a compact compound nucleus is formed [2-4]. The probability of quasifission $\left(P_{Q F}\right)$ can be very large, thus the complementary probability of compound nucleus formation $\left(P_{C N}=1-P_{Q F}\right)$ can be lower than $10^{-3}$ in unfavourable cases. Understanding the competition between quasifission and fusion is thus very important in predicting the optimal fusion reactions to use to form new elements and isotopes in the superheavy mass region.

A key quantity characterizing the non-equilibrium quasifission process is the "sticking time" between contact of the two nuclear surfaces [5] and breakup (scission). Since the two colliding nuclei always approach each other along the beam axis, and after contact rotate

\footnotetext{
a e-mail: david.hinde@anu.edu. au

${ }^{\mathrm{b}}$ Current address: BARC, Mumbai, India

${ }^{\mathrm{c}}$ Permanent address: Department of Physics, School of Mathematical and Physical Sciences, Central University of Kerala, Kasaragod 671314, India

${ }^{\mathrm{d}}$ Current address: National Superconducting Cyclotron Laboratory, Michigan State University, East Lansing, Michigan 48824, USA

${ }^{\text {e }}$ Current address: Malmö University, Faculty of Technology and Society, 20506 Malmö, Sweden
}

(C) The Authors, published by EDP Sciences. This is an Open Access article distributed under the terms of the Creative Commons Attribution License 4.0 (http://creativecommons.org/licenses/by/4.0/). 

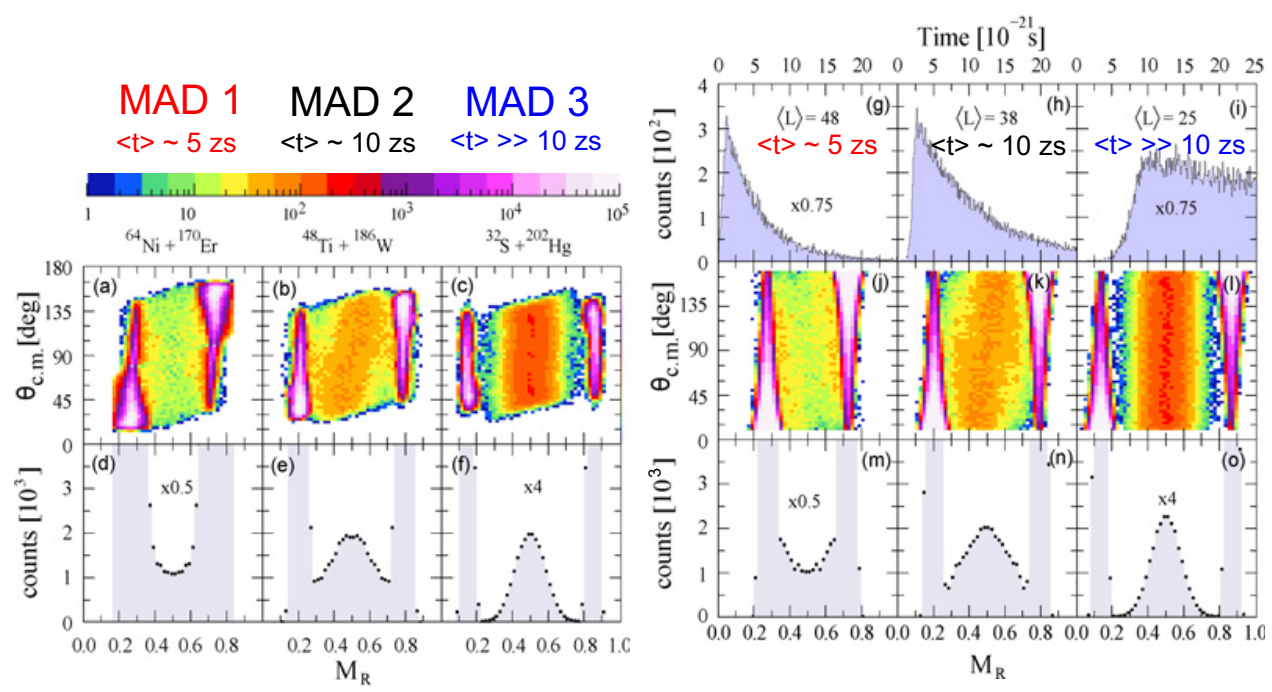

Figure 1. Panels (a)-(c) show the experimental MADs, with (d)-(f) showing the projections onto $M_{R}$, for the reactions ${ }^{64} \mathrm{Ni}+{ }^{170} \mathrm{Er},{ }^{48} \mathrm{Ti}+{ }^{186} \mathrm{~W}$ and ${ }^{32} \mathrm{~S}+{ }^{202} \mathrm{Hg}$, which all form ${ }^{234} \mathrm{Cm}$. The multiplicative factors shown scale the $y$-axis. The simulated MADs for same reactions and beam energies are shown in panels $(\mathrm{j}),(\mathrm{k}),(\mathrm{l})$, with the corresponding $M_{R}$ spectra in panels $(\mathrm{m}),(\mathrm{n}),(\mathrm{o})$, and the sticking time distributions used for the simulations in $(\mathrm{g}),(\mathrm{h}),(\mathrm{i})$. The calculated mean angular momentum following capture, and the deduced approximate mean sticking times are shown in panels (g),(h),(i) (adapted from Ref. [12]).

with angular velocities that can be calculated, measurement of the rotation angle allows estimation of the sticking time. As the system rotates, mass flow also occurs between the two nuclei. A measurement of both mass and angle, for quasifission events over a wide range of angles, is called a mass-angle distribution (MAD). This gives the most direct information on the dynamics, as long as the system undergoes less than a full rotation (taking $\sim 10^{-20} \mathrm{~s}$ ). This is usually the case for collisions of heavy nuclei, as shown by first measurements at GSI $[2,6]$, and later results from ANU [3, 4, 7-13]. The kinematic coincidence technique used in the measurements $[2,3,14]$ provides direct information on the mass-ratio of the fragments at scission; thus, the data are represented in terms of mass ratio $M_{R}$ at scission, rather than pre- or post-evaporation masses of the fragments themselves, which cannot be uniquely determined by this method.

Measured MAD and deduced quasifission sticking time distributions are shown in Fig. 1, for reactions forming the compound nucleus ${ }^{234} \mathrm{Cm}$ [3]. The MAD can be categorised by their distinctly different characteristics: minimum mass yield at symmetry associated with short sticking times; a mass-angle correlation with peak yield at symmetry; and no significant massangle correlation associated with long sticking times. These are assigned to the categories MAD1, MAD2 and MAD3 respectively [3]. It is clear that there is a strong correlation between the MAD type and the entrance channel charge product. However, for particular cases, we have found that detailed entrance channel properties are extremely important in determining the sticking times and MAD characteristics, including neutron richness $[11,15]$, and shell structure including static deformation [10] and magic numbers [11]. This multivariate dependence has resulted in a complex picture.

To clarify and improve our quantitative understanding of the role of shell structures in the dynamics of quasifission, we make an analogy with the liquid drop model approach to nuclear masses, in which localized shell effects can be quantified when the underlying smooth 


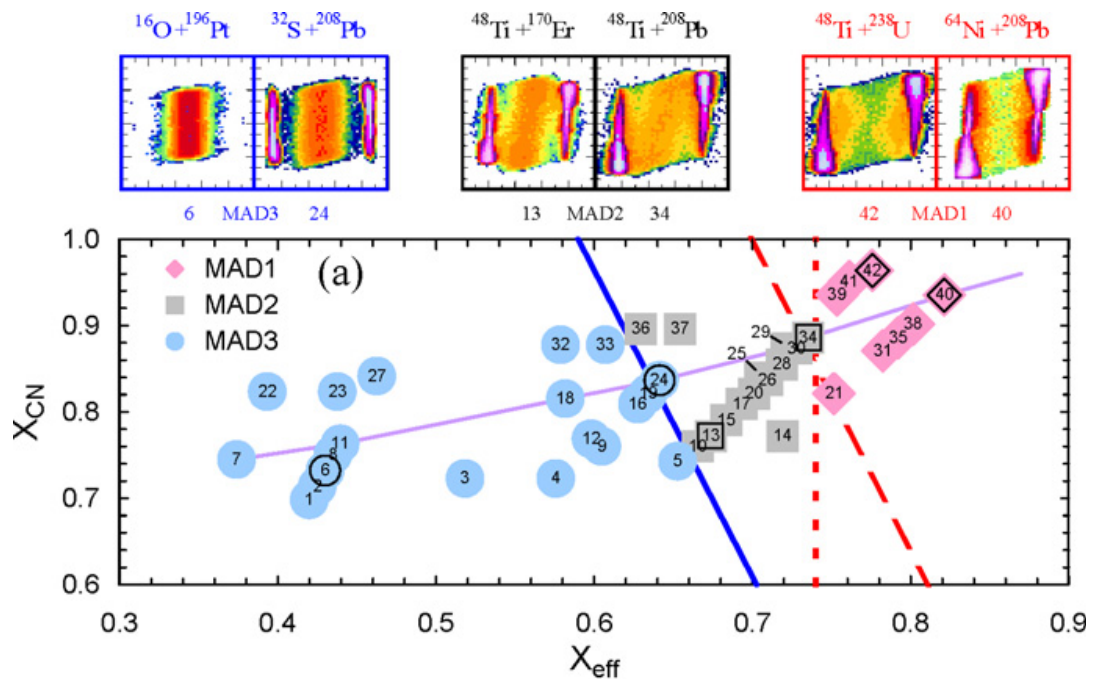

Figure 2. MAD types are plotted as a function of the effective fissility parameter in the entrance channel, $x_{e f f}$, and the compound nucleus fissility parameter, $x_{C N}$ (see text). The numbers inside the symbol indicating the classification of MAD observed refer to the reaction number in Ref. [3]. The diagonal blue line represents the empirical boundary between MAD1 reactions with no mass-angle correlation (left) and MAD2 that have (right). The dashed line indicates the estimated boundary of MAD1 reactions, which show no peak at mass-symmetry in the angle-integrated fission mass distribution. The thin line represents the locus of reactions with ${ }^{208} \mathrm{~Pb}$. Examples of MAD are shown in the panels above, identified by their reaction number.

(liquid drop) trends are well defined. To define the smooth trends in quasifission dynamics, a large number of MAD measurements have been selected, at beam energies somewhat above the capture barrier (typically by $\sim 6 \%$ ). Here the known effects of deformation alignment $[9,10,14,16]$ and shell structure observed in measurements at below-barrier energies $[11,17]$ are much reduced $[10,18,19]$. However, the beam energies should not be too far above the capture barriers, otherwise high angular momenta would be introduced in the collisions, which would then not be representative of heavy element formation reactions.

Using these measured MADS, reaction outcomes (defined for simplicity in terms of the MAD types described above) are plotted against the variables that reflect the balance between nuclear and Coulomb forces during the collision, which are expected to determine a smooth dependence of outcomes of reactions forming very heavy elements. Reference [20] proposed that there should be scaling behavior between reactions, based on the schematic "chaotic regime dynamics" model of fusion of heavy nuclei, which couples shape changes of the nuclear system to internal nucleonic degrees of freedom (one-body dissipation).

The experimental MAD types are plotted as a function of two scaling parameters expected to most strongly define the reaction outcome: (i) the effective fissility parameter of the entrance channel, $x_{e f f}$, and (ii) the fissility parameter of the compound nucleus, $x_{C N}$. The former is most relevant to necked shapes close to the (generally) mass-asymmetric contact configuration in the entrance channel, whilst the latter is applicable to shapes without a constricted neck [21], and where the mass-asymmetry degree of freedom is not constrained.

Figure 2 shows the experimental MAD type as a function of these two variables, with a clear clustering of reactions of each type. The boundary across which a mass-angle correlation becomes significant (between MAD classes 3 and 2) shows a dependence on both variables, with a stronger dependence on the entrance-channel than on the compound nucleus fissility. 


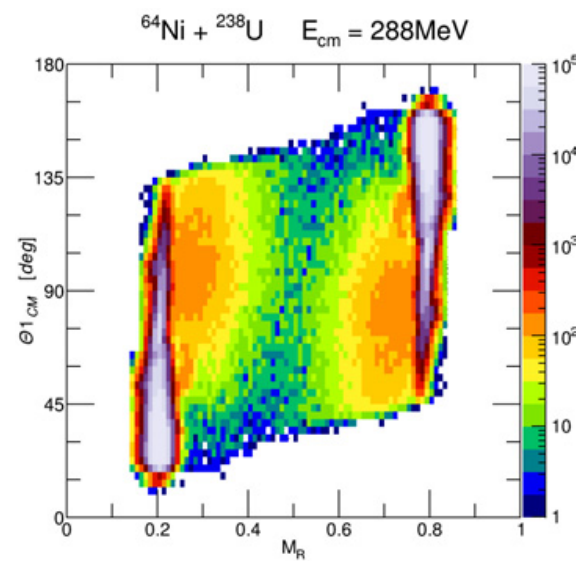

Figure 3. Preliminary mass-angle distribution for the ${ }^{64} \mathrm{Ni}+{ }^{238} \mathrm{U}$ reaction, a candidate reaction to form element 120, measured at an above-barrier energy. This shows a strong mass-angle correlation indicating that typically the sticking time for this reaction is short, with limited mass evolution towards mass-symmetry. Recent TDHF calculations for this reaction [25] predict quasifission to be the outcome, with similar characteristics to those measured. It can also be seen that measurement over a wide angular range is necessary even to get a less-than-complete picture of the total mass-ratio spectrum. Masssymmetric events peak around $90^{\circ}$, but could well be associated with the tail of the quasifission mass distribution. Measurement at angles closer to the beam axis should give greater sensitivity to a fusionfission component.

The full blue line is our estimate of this boundary based on the current data. Mass-angle distributions for reactions on this line should show similar mass-angle correlations, associated with similar reaction trajectories and timescales. The same should be true for reactions on nearby parallel lines. The equation of this boundary line is $0.75 x_{\text {eff }}+0.25 x_{C N}=0.68$, giving $x_{e f f}$ three times the weight of $x_{C N}$. In terms of the MAD, this defines a mean fissility parameter $x_{m}$ which seems to be a good predictor of the quasifission characteristics observed at the higher (above-barrier) beam and excitation energies selected here. The thin full line almost orthogonal to the others represents the locus of reactions with the doubly-magic ${ }^{208} \mathrm{~Pb}$. At the above-barrier energies chosen, no significant departure from the systematic behaviour is seen for reactions on this line.

The behaviour is very different at sub-barrier energies. Here it is well-known that collisions with the tips of prolate deformed nuclei are those that result in capture [5], due to the lower capture barrier for tip collisions. Fission angular distributions [14], mass distributions $[9,22,23]$ and $\operatorname{MAD}[10,13]$ point to the changing nature (shorter sticking time) of quasifission under these circumstances. Microscopic time-dependent HartreeFock (TDHF) calculations of quasifission masses and angles give a good match [13] to experimental results for the reaction ${ }^{40} \mathrm{Ca}+{ }^{238} \mathrm{U}$, including the transition from sub-barrier to above-barrier energies (at which all collision orientations contribute). This is also consistent with the absence of evaporation residue yields at sub-barrier energies [24]. For very heavy systems, such as the ${ }^{64} \mathrm{Ni}+{ }^{238} \mathrm{U}$ reaction, even at an above-barrier energy, the MAD (shown in Fig. 3) indicates that the majority of capture reactions result in rapid re-separation, with limited mass evolution towards symmetry. Extending the experimental MAD to angles even closer to the beam axis would increase sensitivity to a fusion-fission component (expected close to mass-symmetry), as the quasifission events at this energy are concentrated close to $90^{\circ}$. 


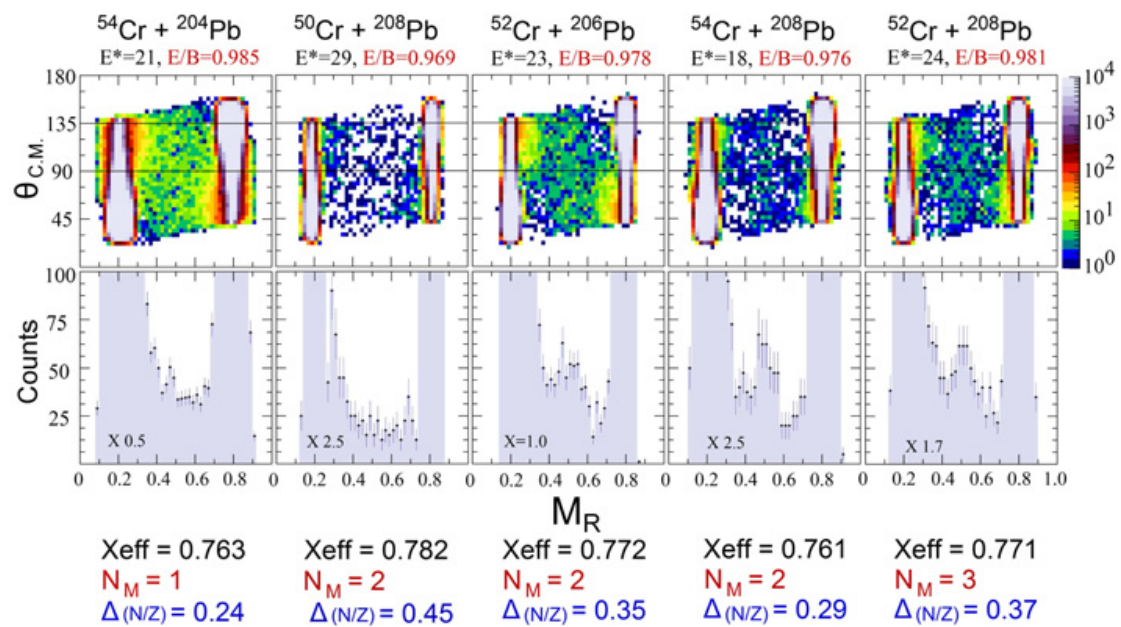

Figure 4. MAD and projected mass-ratio distributions for backward angles from $90^{\circ}$ to $135^{\circ}$ (as indicated in the MAD plots), for reactions of ${ }^{50,52,54} \mathrm{Cr}$ isotopes with ${ }^{204,206,208} \mathrm{~Pb}$. Sub-barrier beam energies (denoted by $E / B$ ), resulted in the low excitation energies $E^{*}$. The entrance channel fissility, $x_{e f f}$, the number of entrance-channel magic numbers, and the mismatch in $N / Z$ values are indicated for each reaction (see text). The reaction outcome changes from a minimum in yield at mass-symmetry (left) to a narrow peak at symmetry, having no evidence of a mass-angle correlation.

Sub-barrier reactions with (spherical) closed-shell nuclei show contrasting behaviour to reactions with heavy statically deformed nuclei. It has been shown [11] that increasing the "magicity" in the entrance channel at sub-barrier energies results in narrower mass distributions, correlated with a reduced mass-angle correlation, indicative of longer sticking times. Indeed, for the ${ }^{48} \mathrm{Ca}+{ }^{208} \mathrm{~Pb}$ reaction, measured mass distributions [17] appear consistent with a fusion-fission mechanism. These results led to the conclusion that "magicity" plays its strongest role when the $N / Z$ values of the projectile and target nuclei are matched. When this is the case, transfer reactions that destroy entrance-channel magicity (as in the ${ }^{40} \mathrm{Ca}+{ }^{208} \mathrm{~Pb}$ reaction [11]) are minimized, preserving the closed shell nature of the collision partners as long as possible during the merging of the two nuclei [11].

Recent ANU results, for the reaction of isotopes of $\mathrm{Cr}$ with $\mathrm{Pb}$, are shown in Fig. 4. The left three reactions all form the compound nucleus ${ }^{258} \mathrm{Sg}$. These measurements were at sub-barrier energies, resulting in the low excitation energies (above the ground-state) as indicated. They support the picture from the $\mathrm{Ca}+\mathrm{Pb}$ data. The difference between the $N / Z$ values of the target and projectile nuclei is denoted by $\Delta_{N / Z}$ in the figure. The panels are ordered from left to right first by the number of magic numbers in the entrance channel $\left(N_{M}\right)$, and then by $\Delta_{N / Z}$. The left-most reaction has only a single magic number in the entrance channel, and shows a U-shaped mass distribution, consistent with MAD class 1 . With two magic numbers, the reactions better matched in $N / Z$ ( $\Delta_{N / Z}$ closer to zero) show a peak at mass-symmetry, associated with an angle-independent ridge in the MAD. With three magic numbers, but less favourable $\Delta_{N / Z}$, a similar result is observed. As the beam energy is increased, this mass-symmetric peak becomes a smaller and smaller fraction of the total fission yield within the range $0.3<M_{R}<0.7$. These reactions show a very similar behaviour to the ${ }^{40,44,48} \mathrm{Ca}+{ }^{204,208} \mathrm{~Pb}$ reactions; however, the transition from a U-shaped mass distribution to a narrow mass-symmetric component in the mass distribution is in some ways an even more drastic change in reaction outcome. The very sudden change in 
outcome, depending on neutron number and magicity, will be a severe challenge for models of quasifission to reproduce. And yet it is this level of sensitivity that models must strive to reproduce, to optimise experimental opportunities to create new isotopes of superheavy elements. Investigating the relationship between these changing quasifission characteristics and changes in the superheavy element yield would be of great value.

\section{References}

[1] D.J. Hinde, M. Dasgupta, A. Mukherjee, Phys. Rev. Lett. 89, 282701 (2002).

[2] J. Tōke et al., Nucl. Phys. A440, 327 (1985).

[3] R. du Rietz et al., Phys. Rev. C 88, 054618 (2013).

[4] R. du Rietz et al., Phys. Rev. Lett. 106, 052701 (2011).

[5] M. Dasgupta, D.J. Hinde, N. Rowley, A.M. Stefanini, Annu. Rev. Nucl. Part. Sci. 48, 401 (1998).

[6] W.Q. Shen et al., Phys. Rev. C 36, 115 (1987).

[7] R.G. Thomas et al., Phys. Rev. C 77, 034610 (2008).

[8] R. Rafiei et al., Phys. Rev. C 77, 024606 (2008).

[9] D.J. Hinde, R. du Rietz, M. Dasgupta, R.G. Thomas, L.R. Gasques, Phys. Rev. Lett. 101, 092701 (2008).

[10] D.J. Hinde et al., Phys. Rev. Lett. 100, 202701 (2008).

[11] C. Simenel et al., Phys. Lett. B710, 607 (2012).

[12] E. Williams et al., Phys. Rev. C 88, 034611 (2013).

[13] A. Wakhle et al., Phys. Rev. Lett. 113, 182502 (2014).

[14] D.J. Hinde et al., Phys. Rev. C 53, 1290 (1996).

[15] K. Hammerton et al., Phys. Rev. C 91, 041602 (2015).

[16] D.J. Hinde et al., Phys. Rev. Lett. 74, 1295 (1995).

[17] E.V. Prokhorova et al., Nucl. Phys. A802, 45 (2008).

[18] K. Nishio et al., Phys. Rev. C 77, 064607 (2008).

[19] I.M. Itkis et al., Phys. Rev. C 83, 064613 (2011).

[20] J.P. Blocki, H. Feldmeier, W.J. Swiatecki, Nucl. Phys. A459, 145 (1986).

[21] S. Bjørnholm and W.J. Swiatecki, Nucl. Phys. A391, 471 (1982).

[22] C.J. Lin et al., Phys. Rev. C 85, 014611 (2012).

[23] K. Nishio et al., Phys. Rev. C 82, 044604 (2010).

[24] S. Mitsuoka, H. Ikezoe, K. Nishio, J. Lu, Phys. Rev. C 62, 054603 (2000).

[25] K. Sekizawa and K. Yabana, Phys. Rev. C 93, 054616 (2016). 